\title{
Novel piperazine substituted indole derivatives: Synthesis, anti-inflammatory and antioxidant activities and molecular docking
}

\author{
Tunca Gül ALTUNTAŞ 1 (D), Aziz BAYDAR 1 (D), Zühal KILIÇ-KURT ${ }^{1}$ * (D) Cemre ACAR ${ }^{1}$ (D), \\ Sezen YILMAZ-SARIALTIN 2 (D), Tülay ÇOBAN 2 (D) \\ 1 Department of Pharmaceutical Chemistry, Faculty of Pharmacy, Ankara University, Ankara, Turkey. \\ 2 Department of Pharmaceutical Toxicology, Faculty of Pharmacy, Ankara University, Ankara, Turkey. \\ * Corresponding Author. E-mail: zkurt@ankara.edu.tr (Z.K.K.); Tel. +90-312-203 3073.
}

Received: 07 November 2019 / Revised: 02 April 2020/ Accepted: 09 April 2020

ABSTRACT: In this work, a series of piperazine substituted indole derivatives were synthesized and evaluated for their in vitro antioxidant and anti-inflammatory activities. The results of antioxidant activity showed that compounds 2 $(81.63 \%)$ and $11(85.63 \%)$ had comparable DPPH free radical scavenging activity to Vit E (88.6\%). The in vitro antiinflammatory assays indicated that most of the compounds had more higher anti-inflammatory activities than standart ASA. Docking results revealed that compound 11 possessing the strongest anti-inflammatory activities showed the Hbond interactions with the key residues of COX-2 active site. It suggested that the anti-inflammatory activity of the compounds might result from COX-2 inhibition. It will be verified with further enzyme inhibition assays.

KEYWORDS: Anti-inflammatory activity; antioxidant activity; COX-2; piperazine; indole.

\section{INTRODUCTION}

Inflammation is a cellular protective response against a various triggering factors such as infectious agents, foreign pathogens, free radicals, tissue damage and cellular injury. Exaggerated and prolonged inflammation which seriously threatens human health may lead to tissue damage and various chronic diseases such as atherosclerosis, rheumatoid arthritis, sepsis, psoriasis, prostatitis, alzheimer and cancer [1,2]. Many enzymes, reactive oxygen, nitrogen and chlorine species and chemical mediators are released during the infiltration of inflammatory cells, and oxidative stress is induced[3]. Production of reactive species could initiate inflammation by activating multiple pathways such as redox-sensitive transcription factors including nuclear factor-kappaB (NF-kB) and activator protein-1 (AP-1). The reactive oxygen species (ROS) also is involved in conversion of arachidonic acid into proinflammatory intermediates and prostaglandins through cyclooxygenase-1 (COX-1) and lipoxygenase (LOX) [4-6]. Several studies show that inflammation and oxidative stress are related with each other in the development of many chronic disease such as diabetic complications [7,8] cardiovascular [9] and neurodegenerative diseases, [10,11] alcoholic liver disease, [12] and chronic kidney disease [13,14].

Indole compounds are one of the most studied heterocyclic scaffolds in medicinal chemistry because of their wide range of bioactivities such as anti-inflammatory, anti-viral, anti-HIV, anti-depressant, antihistaminic, anti-hypertensive, and anti-diabetic $[15,16]$. To date only a few indole-based anti-inflammatory agents have been reported. Indomethacin (Figure 1), approved by Food and Drug Administration (FDA) in 1965 as a non-selective inhibitor of COX-1 and COX-2. It is used to reduce fever, pain and swelling by inhibiting the production of prostaglandins $[17,18]$. Tenidap (Figure 1) was developed as a COX/5-LOX inhibitor which have cytokine modulating anti-inflammatory and anti-rheumatoid activity. But, it was rejected due to its liver and kidney toxicity [19]. Acemetacin (Figure 1), [20] a prodrug of indomethacin, and etodolac (Figure 1), [21] a selective COX-2 inhibitor, are used for treatment of osteoarthritis and rheumatoid arthritis. Besides the anti-inflammatory effects of indomethacin, etodolac and acemetacin, they also have ROS and the reactive nitrogen species (RNS) scavenging activity [22]. Significant indole compounds such as melatonin, [23] tryptophan, serotonin, indole alkaloids, indole-3-acetic acid [24] and other synthetic derivatives [25-32] show

How to cite this article: Altuntaş TG, Baydar A, Kılıç-Kurt Z, Acar C, Yılmaz-Sarıaltın S, Çoban T. Novel piperazine substituted indole derivatives: Synthesis, anti-inflammatory and antioxidant activities and molecular docking. J Res Pharm. 2020; 24(3): 350-360. 
antioxidant potential by ROS and RNS scavenge ability. On the basis of the underlying mechanism of several chronic diseases, development of compounds inhibiting both oxidative stress and inflammation might be considered as an efficient approach for treatment of many diseases.<smiles>COc1ccc2c(c1)c(CC(=O)O)c(C)n2C(=O)c1ccc(Cl)cc1</smiles>

Indomethacine<smiles>NC(=O)N1C(=O)/C(=C(/O)c2cccs2)c2cc(Cl)ccc21</smiles>

Tenidap<smiles>COc1ccc2c(c1)c(CC(=O)OCC(=O)O)c(C)n2C(=O)c1ccc(Cl)cc1</smiles>

Acemetacin<smiles>CCc1cccc2c3c([nH]c12)C(CC)(CC(=O)O)OCC3</smiles>

Etodolac

Figure 1. Indole based anti-inflammatory drugs.

We have previously reported the synthesis and in vitro antioxidant activity of a series of non substitue indole compounds bearing piperazine derivatives. They showed significant superoxide anion scavenging activity (88-69\%) at $1 \mathrm{mM}$ concentration [33]. In order to compare the activity, in this work, novel 5-methoxy and 5-fluoro indole derivatives (Figure 2) containing substituted-phenyl piperazine moiety at 2-position were synthesized. Their antioxidant and anti-inflammatory activity were evaluated. In addition, molecular docking of the most active compounds into COX-2 enzyme was performed by using Autodock vina.

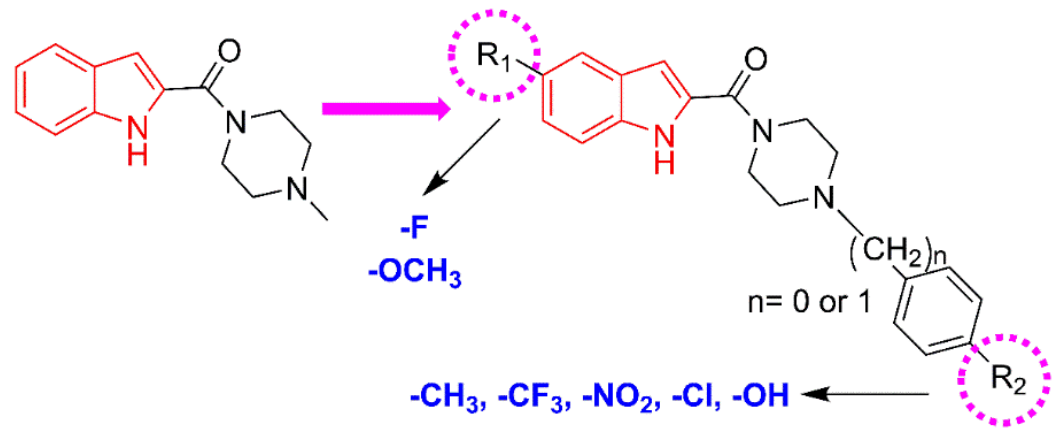

Figure 2. Designing of new 5-methoxy and 5-fluoro indole derivatives.

\section{RESULT AND DISCUSSION}

\subsection{Chemistry}

The synthesis of the target compounds containing substituted-piperazine derivatives is outlined in Figure 3. Compound 1-11 were obtained by reacting 5-substituted-indole-2-carboxylic acid with appropriate piperazine derivatives in the presence of carbonyldiimidazole (CDI) in anhydrous tetrahydrofuran (THF) under nitrogen atmosphere [34]. The structures of all synthesized compounds were characterized by ${ }^{1} \mathrm{H}$ NMR, ${ }^{13} \mathrm{C}$ NMR, MASS and elementel analysis. In the ${ }^{1} \mathrm{H}$ NMR spectra, the signal of the piperazine protons was observed as two broad singlet or triplet at around 2.41-3.61 ppm and 3.72-3.93 ppm for each molecule. NH proton of indole ring was observed as a characteristic singlet, which ranged from 11.38 to $11.72 \mathrm{ppm}$. H-3 proton of indole ring was detected between 6.67-6.84 ppm for each molecule. In the spectrum of the compounds 7 and 8 , a singlet at 3.49 and $3.50 \mathrm{ppm}$ was observed due to the $\mathrm{CH}_{2}$ protons, respectively.

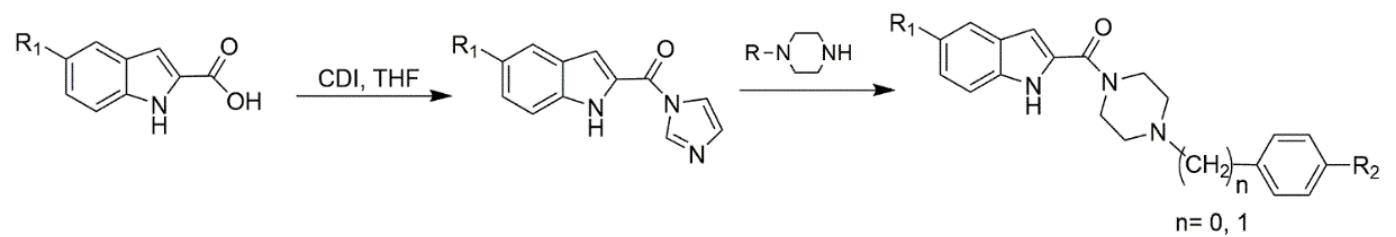

Figure 3. Synthesis of indole containing piperazine derivatives (1-11). 


\subsection{Biological activity}

The target compounds were evaluated for their antioxidant activity by using different methodologies such as 2,2-diphenyl-1-picrylhydrazyl (DPPH) stable radical and superoxide anion scavenging activity and lipid peroxidation (LP) inhibition. The obtained results were compared with the standard antioxidant vitamin $\mathrm{E}$ (Vit-E) in Table 1. Compounds 2 (81.63\%) and $\mathbf{1 1}$ (85.63\%) showed the highest DPPH free radical scavenging activity at $0.1 \mathrm{mM}$ concentration, respectively. All compounds, except 2 and 11, displayed no scavenging effect on LP and superoxide anion at 0.1 and $0.01 \mathrm{mM}$ concentrations. Compound $\mathbf{2}$ had strong superoxide anion scavenging activity (51\%) and comparable LP inhibition (46\%) with vit-E (51\%) at $0.1 \mathrm{mM}$. Better LP inhibition activity (79\%) than vit-E (51\%) was observed with compound 11 at $0.1 \mathrm{mM}$. Otherwise, compound 11 exhibited low superoxide anion scavenging activity (22\%). Compounds 5, 8 and 10 showed no inhibition effect on all assay.

Table 1. Antioxidant activities of the newly synthesized compounds (1-11).

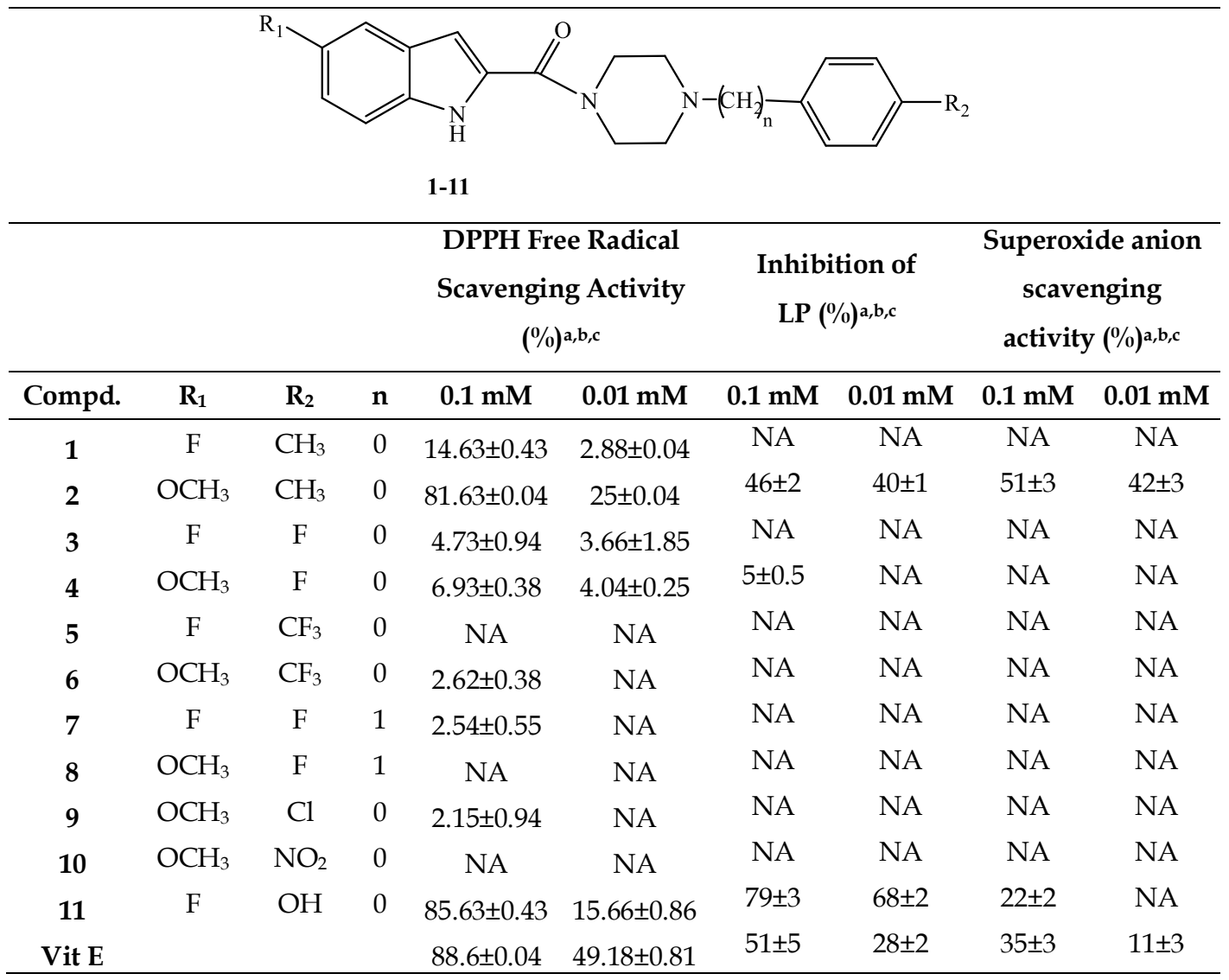

aThe values represent the average of 3 determinations $\pm S D$.

bCompounds were diluted with DMSO (solvent showed no antioxidant activity).

${ }^{c} \mathrm{p}<0.05$ vs. DMSO by ANOVA/Tukey's test. NA; no activity.

The human red blood cell (HRBC) membrane stabilization could be a significant in vitro measure of antiinflammatory activity of the drugs. The membrane stabilizing activities of the newly synthesized compounds (1-11) and previously synthesized compounds (12-26) [33] were evaluated using heat induced human erythrocyte hemolysis in comparison with acetylsalisilic acid (ASA) used as reference drug. Table 2 depicted that 5-substituted indole derivatives (1-11, except compound 4) showed more inhibition of heat induced hemolysis compared to indole derivatives (12-26) as well as ASA. Substitution at 5-position of the indole ring with fluoro and methoxy groups might have contributed to the increased anti-inflammatory potency of the compounds. Replacement of fluoro $\left(1, \mathrm{IC}_{50}=1.19 \mathrm{mM}\right)$ with methoxy $\left(2, \mathrm{IC}_{50}=0.68 \mathrm{mM}\right)$ at 5-position of indole ring, resulted in 1.7-fold increase in membrane stabilizing activity. In contrast, the same methoxy substitution at 5-position of indole ring decreased the activity of compounds 4, 6 and 8 relative to compound $\mathbf{3}, \mathbf{5}$ and 7. Replacing the hydroxyl group at $4^{\prime}$-position of phenyl piperazine moiety $\left(\mathbf{1 1}, \mathrm{IC}_{50}=0.33 \mathrm{mM}\right)$ with fluoro $\left(3, \mathrm{IC}_{50}=0.64 \mathrm{mM}\right)$ and methyl $\left(\mathbf{1}, \mathrm{IC}_{50}=1.19 \mathrm{mM}\right)$ caused a dramatic loss in membrane stabilization 
activity, while the trifluoromethyl group $\left(5, \mathrm{IC}_{50}=0.43 \mathrm{mM}\right)$ is well tolerated. In case of 5-methoxy indole derivatives, the nitro group at $4^{\prime}$-position of phenyl piperazine exhibited stronger membrane stabilization activity $\left(\mathbf{1 0}, \mathrm{IC}_{50}=0.40 \mathrm{mM}\right)$ than corresponding derivatives bearing chloro (9), $\mathrm{CF}_{3}(6)$, fluoro (4) and methyl (2). The compounds $\mathbf{2}$ and $\mathbf{1 1}$ were found 2- and 4.3-fold more active than ASA as anti-inflammatory activity which showed the correlation with antioxidant activity of them. Although 5-methoxy substitution on indole ring resulted in 5.8-fold increase in membrane stabilization activity of compound $\mathbf{1 0}$ compared to corresponding indole derivative $\mathbf{2 1}$, it did not lead to significantly change in membrane stabilization activity of the compounds 4 and 22.

Table 2. Human red blood cell membrane stabilizing effects of the compounds.
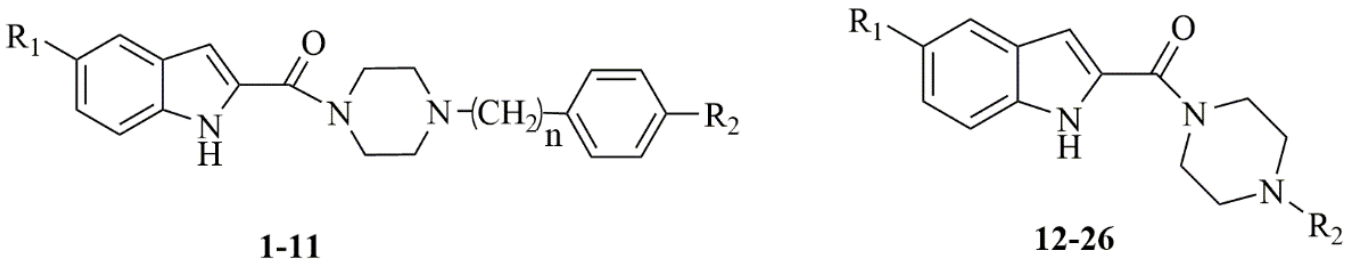

\begin{tabular}{|c|c|c|c|c|c|c|c|c|c|}
\hline Compd.a & $\mathbf{R}_{\mathbf{1}}$ & $\mathbf{R}_{2}$ & $\mathbf{n}$ & $\begin{array}{c}\mathrm{IC}_{50} \pm \mathrm{SD} \\
(\mathrm{mM})\end{array}$ & Compd.a & $\mathbf{R}_{\mathbf{1}}$ & $\mathbf{R}_{\mathbf{2}}$ & $\mathbf{n}$ & $\begin{array}{c}\mathrm{IC}_{50} \pm \mathrm{SD} \\
(\mathrm{mM})\end{array}$ \\
\hline 1 & $\mathrm{~F}$ & $\mathrm{CH}_{3}$ & 0 & $1.19 \pm 0.05^{*}$ & 14 & $\mathrm{H}$ & 3-nitro-2-pyridyl & 0 & $2.21 \pm 0.03^{*}$ \\
\hline 2 & $\mathrm{OCH}_{3}$ & $\mathrm{CH}_{3}$ & 0 & $0.68 \pm 0.01^{*}$ & 15 & $\mathrm{H}$ & $\mathrm{CH}_{3}$ & 0 & $1.95 \pm 0.01^{*}$ \\
\hline 3 & $\mathrm{~F}$ & $\mathrm{~F}$ & 0 & $0.64 \pm 0.01^{*}$ & 16 & $\mathrm{H}$ & $\mathrm{CH}_{2} \mathrm{CH}_{2} \mathrm{OH}$ & 0 & $1.83 \pm 0.05^{*}$ \\
\hline 4 & $\mathrm{OCH}_{3}$ & $\mathrm{~F}$ & 0 & $3.11 \pm 0.02^{*}$ & 17 & $\mathrm{H}$ & 3-chlorobenzyl & 0 & $2.54 \pm 0.01^{*}$ \\
\hline 5 & F & $\mathrm{CF}_{3}$ & 0 & $0.43 \pm 0.01^{*}$ & 18 & $\mathrm{H}$ & diphenylmethyl & 0 & $2.76 \pm 0.06^{*}$ \\
\hline 6 & $\mathrm{OCH}_{3}$ & $\mathrm{CF}_{3}$ & 0 & $0.54 \pm 0.02 *$ & 19 & $\mathrm{H}$ & cyclohexyl & 0 & $2.38 \pm 0.08^{*}$ \\
\hline 7 & F & $\mathrm{F}$ & 1 & $0.46 \pm 0.04^{*}$ & 20 & $\mathrm{H}$ & phenyl & 0 & $5.45 \pm 0.09^{*}$ \\
\hline 8 & $\mathrm{OCH}_{3}$ & $\mathrm{~F}$ & 1 & $0.68 \pm 0.02^{*}$ & 21 & $\mathrm{H}$ & $p$-nitrophenyl & 0 & $2.34 \pm 0.03^{*}$ \\
\hline 9 & $\mathrm{OCH}_{3}$ & $\mathrm{Cl}$ & 0 & $0.56 \pm 0.01^{*}$ & 22 & $\mathrm{H}$ & $p$-fluorophenyl & 0 & $3.84 \pm 0.02 *$ \\
\hline 10 & $\mathrm{OCH}_{3}$ & $\mathrm{NO}_{2}$ & 0 & $0.40 \pm 0.03^{*}$ & 23 & $\mathrm{H}$ & pyrimidine-2-yl & 0 & $2.32 \pm 0.07^{*}$ \\
\hline 11 & $\mathrm{~F}$ & $\mathrm{OH}$ & 0 & $0.33 \pm 0.02^{*}$ & 24 & $\mathrm{H}$ & acetyl & 0 & $1.97 \pm 0.01^{*}$ \\
\hline 12 & $\mathrm{H}$ & $\mathrm{CHO}$ & 0 & $2.32 \pm 0.01^{*}$ & 25 & $\mathrm{H}$ & cyclopropylcarbonyl & 0 & $1.73 \pm 0.02 *$ \\
\hline 13 & $\mathrm{H}$ & $\mathrm{H}$ & 0 & $2.27 \pm 0.02^{*}$ & 26 & $\mathrm{H}$ & ethoxycarbonyl & 0 & $2.30 \pm 0.02^{*}$ \\
\hline ASA & & & & $1.42 \pm 0.03^{*}$ & & & & & \\
\hline
\end{tabular}

a Synthesis of compound 12-26 was previously reported in lit. 33. ( $\left.{ }^{*}\right)$ Statistically significant as compared to control. p<0.05.

\subsection{Molecular docking and prediction of molecular properties}

In order to understand whether the anti-inflammatory activity of the compounds is correlated with COX-2 (PDB code: 3NT1) enzyme inhibition, the molecular docking study was performed using Autodock vina. Firstly, the validation of the docking procedure was done by docking of crystallographic naproxen over 3NT1. The docked naproxen is superimposed on crystallographic naproxen forming two hydrogen bonds with Arg120 and one hydrogen bond with Tyr355 and RMSD value was found as 0.702 A (Figure 4, left) [35]. Compound 11 possessing the best anti-inflammatory activity formed a hydrogen bond between amide carbonyl and NH of Arg120 at the distance of $2.23 \AA$ (Figure 4, right). The docking results suggested that the anti-inflammatory activities of the compound $\mathbf{1 1}$ might correlate with its COX-2 interactions. In order to verify the results of molecular docking and present the mechanism of anti-inflammatory activity, further COX-2 enzyme inhibition studies will be performed.

Molecular properties of the synthesized compounds (1-11) were calculated using online Molinspiration property program [36]. The predicted volume, topological polar surface area (TPSA), number of violations and Lipinski parameters [37] such as molecular weight (MW), number of rotatable bonds ( $n$ ROTB), number of hydrogen bond acceptors $(n \mathrm{ON})$ number of hydrogen bond donors $(n \mathrm{OHNH})$, and lipophilicity $(\operatorname{miLogP})$ were calculated. As shown in Table 3, all compounds confirm the Lipinski's rules with $\log P$ values ranged from 2.11-4.35, MW ranging from 339.40-403.40, HBA value of $\leq 10$, HBD value of $\leq 5$ and $n$ ROTB values of $<10$. 
The percentage of absorption (\%ABS) is calculated by using $\%$ ABS $=109-(0.345 \times$ TPSA $)$ [38]. All compounds (except compound 10) have the good $\mathrm{ABS} \%$ value of $>80 \%$, suggesting that the synthesized compounds provide acceptable flexibility and favorable permeability and oral bioavailability.

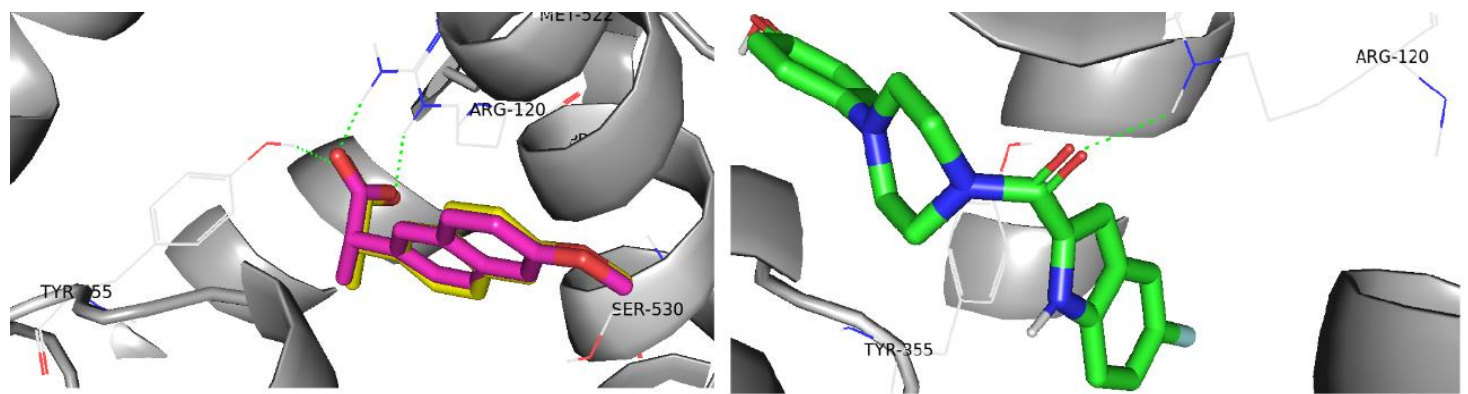

Figure 4. The superimposition of crystal naproxen (yellow) and docked naproxen (magenta) (left). The predicted binding mode of compound $\mathbf{1 1}$ (green) in the catalytic site of COX-2 (pdb code: 3NT1) are generated by the programme PyMOL (right). The hydrogen bonds are presented by dotted green lines and residues forming hydrogen bonds with ligand are shown in element coloured line.

Table 3. Prediction of molecular properties parameters and drug-likeness scores of indole containing piperazine derivatives (1-11).

\begin{tabular}{cccccccccc}
\hline Cpd & MWa & Volume & \%ABSb & TPSAc & $n$ ROTBd & $n$ ONe & $n$ OHNH $^{f}$ & LogPg & $n$ violations \\
\hline Rule & $<500$ & - & - & - & $<10$ & $\leq 10$ & $\leq 5$ & $\leq 5$ & $\leq 1$ \\
$\mathbf{1}$ & 337.40 & 306.84 & 95.43 & 39.34 & 2 & 4 & 1 & 3.04 & 0 \\
$\mathbf{2}$ & 349.43 & 327.45 & 92.25 & 48.57 & 3 & 5 & 1 & 3.91 & 0 \\
$\mathbf{3}$ & 341.36 & 295.21 & 95.43 & 39.34 & 2 & 4 & 1 & 2.76 & 0 \\
$\mathbf{4}$ & 353.40 & 315.82 & 92.25 & 48.57 & 3 & 5 & 1 & 3.62 & 0 \\
$\mathbf{5}$ & 391.37 & 321.57 & 95.43 & 39.34 & 3 & 4 & 1 & 3.49 & 0 \\
$\mathbf{6}$ & 403.40 & 342.19 & 92.25 & 48.57 & 4 & 5 & 1 & 4.35 & 0 \\
$\mathbf{7}$ & 355.39 & 312.01 & 95.43 & 39.34 & 3 & 4 & 1 & 2.46 & 0 \\
$\mathbf{8}$ & 367.42 & 332.62 & 92.25 & 48.57 & 4 & 5 & 1 & 3.32 & 0 \\
$\mathbf{9}$ & 369.85 & 324.43 & 92.25 & 48.57 & 3 & 5 & 1 & 4.14 & 0 \\
$\mathbf{1 0}$ & 380.40 & 334.23 & 76.43 & 94.40 & 4 & 8 & 1 & 3.42 & 0 \\
$\mathbf{1 1}$ & 339.37 & 298.30 & 88.44 & 59.57 & 2 & 5 & 2 & 2.11 & 0 \\
\hline
\end{tabular}

a MW: Molecular weight; b \% ABS: Percentage absorption; cTPSA: Topological polar surface area; $n$ ROTB: Number of rotatable bonds; $\mathrm{e} n \mathrm{ON}$ : Number of hydrogen acceptors; ${ }^{\mathrm{f}} n \mathrm{OHNH}$ : Number of hydrogen donors; $\mathrm{g}$ LogP: $\log$ octanol/water partition coefficient.

\section{CONCLUSION}

In conclusion, several 5-substituted indole derivatives bearing piperazine group were synthesized for evaluation of their antioxidant and anti-inflammatory activity based on our previously obtained results from corresponding non-substituted indole derivatives [33]. The in vitro antioxidant and anti-inflammatory activity results revealed that the 5-substituted indoles were more active than non-substituted indole derivatives, suggesting substitution at 5-position of indole ring could be important for activity. Compounds $\mathbf{2}$ and $\mathbf{1 1}$ possessing the highest DPPH radical scavenging activity also showed strong anti-inflammatory activity with $\mathrm{IC}_{50}$ value of 0.68 and $0.33 \mathrm{mM}$. Moreover, compound 11 located into active site of COX-2 forming hydrogen bond with Arg120. In the future, whether the strong anti-inflammatory activity of compound $\mathbf{1 1}$ result from COX-2 inhibition will be evaluated.

\section{MATERIALS AND METHODS}

The chemical reagents were purchased from commercial suppliers and used without further purification. The reactions were monitored and the purity of the products was checked by thin layer chromatography (TLC). Merck silica gel $60 \mathrm{~F}_{254}$ chromatoplates were used for TLC. Uncorrected melting points were measured on a Büchi B-540 capillary melting point apparatus. ${ }^{1} \mathrm{H}(400 \mathrm{MHz})$ and ${ }^{13} \mathrm{C}(100 \mathrm{MHz}) \mathrm{NMR}$ spectra were recorded on Varian Mercury $400 \mathrm{MHz}$ FT spectrometer (Agilent Technologies, USA) in DMSO- 
$d_{6}, \mathrm{CDCl}_{3}$, and $\mathrm{CD}_{3} \mathrm{OD}$ as solvent. The chemical shifts $(\delta)$ were recorded in parts per million relative to tetramethylsilane (TMS) and coupling constants $(J)$ are reported in Hertz. The Mass spectra were recorded on a Waters micromass ZQ, using ESI(+). Elemental analysis were carried by Leco-932 CHNS-O analyzer. The results of the elemental analysis $(\mathrm{C}, \mathrm{H}, \mathrm{N})$ were within $\pm 0.4 \%$ of the calculated amounts. Biological assays were carried by using a microplate reader (SpectraMax 190, Molecular Devices, USA).

\subsection{General procedure for the synthesis of compounds}

A solution of CDI in THF ( $3 \mathrm{ml}, 3.7 \mathrm{mmol})$ was added to 5-substituted-indole-2-carboxylic acid (3.1 $\mathrm{mmol})$ in THF $(5 \mathrm{ml})$ at room temperature and stirred for $1 \mathrm{~h}$ under $\mathrm{N}_{2}$ atmosphere. Then, the reaction mixture was cooled to $0^{\circ} \mathrm{C}$ and $\mathrm{N}$-substituted piperazine derivatives $(3.7 \mathrm{mmol})$ in THF ( $\left.3 \mathrm{ml}\right)$ were added and stirred for further 17-18 h at room temperature. Basic work-up $\left(\mathrm{CHCl}_{3}\right.$, sat. $\left.\mathrm{NaHCO}_{3}\right)$ was applied, evaporated under vacuo and recrystallization from ethyl acetate: $n$-hexane provided the desired compounds.

\subsection{1. (5-Fluoro-1H-indol-2-yl)(4-(p-tolyl)piperazin-1-yl)methanone (1)}

CAS Registry Number: 2325638-42-2. Yield: 62\%; mp.: 233-234 ${ }^{\circ} \mathrm{C} .{ }^{1} \mathrm{H}-\mathrm{NMR}\left(400 \mathrm{MHz}, \mathrm{DMSO}-d_{6}\right) ; \delta$ ppm: $2.19\left(\mathrm{~s}, 3 \mathrm{H},-\mathrm{CH}_{3}\right), 3.14(\mathrm{t}, 4 \mathrm{H}$, piperazine- $\mathrm{H}), 3.87(\mathrm{bs}, 4 \mathrm{H}$, piperazine- $\mathrm{H}), 6.81\left(\mathrm{~d}, 1 \mathrm{H}, J_{m}=1.6 \mathrm{~Hz}, \mathrm{H}-3\right)$, $6.86\left(\mathrm{~d}, 2 \mathrm{H}, J_{o}=8.8 \mathrm{~Hz}, \mathrm{H}-2^{\prime}, 6^{\prime}\right), 7.01-7.07(\mathrm{~m}, 3 \mathrm{H}), 7.36\left(\mathrm{dd}, 1 \mathrm{H}, J_{m}=2.8 \mathrm{~Hz}, J_{o}=10 \mathrm{~Hz}\right), 7.41\left(\mathrm{dd}, 1 \mathrm{H}, J_{m}=4.4 \mathrm{~Hz}\right.$, $\left.J_{0}=8.8 \mathrm{~Hz}\right), 11.71(\mathrm{~s}, 1 \mathrm{H}, \mathrm{N}-\mathrm{H}) .{ }^{13} \mathrm{C}-\mathrm{NMR} \delta \mathrm{ppm}\left(\mathrm{DMSO}-d_{6}\right): 19.957 ; 49.043 ; 103.950 ; 105.386 ; 111.795 ; 113.155$; 116.070; 126.787; 128.194; 129.352; 131.539; 132.583; 148.563; 155.893; 158.210; 161.593. MS (ESI +) m/z: 338.3 $(\mathrm{M}+\mathrm{H}, 100 \%)$. Anal. Calcd for $\mathrm{C}_{20} \mathrm{H}_{20} \mathrm{FN}_{3} \mathrm{O} \cdot 0.1 \mathrm{H}_{2} \mathrm{O}: \mathrm{C}, 70.81 ; \mathrm{H}, 6.00 ; \mathrm{N}, 12.38$. Found: $\mathrm{C}, 70.84 ; \mathrm{H}, 6.21 ; \mathrm{N}$, 12.31 .

\subsection{2. (5-Methoxy-1H-indol-2-yl)(4-(p-tolyl)piperazin-1-yl)methanone (2)}

Yield: 53\%; mp.: 203-205 ${ }^{\circ} \mathrm{C} .{ }^{1} \mathrm{H}-\mathrm{NMR}(400 \mathrm{MHz}$, DMSO-d $)$; $\delta$ ppm: 2.25 (s, 3H, $\left.-\mathrm{CH}_{3}\right), 3.20$ (bs, $4 \mathrm{H}$, piperazine-H), $3.80\left(\mathrm{~s}, 3 \mathrm{H},-\mathrm{OCH}_{3}\right), 3.93(\mathrm{bs}, 4 \mathrm{H}$, piperazine-H), $6.79(\mathrm{~s}, 1 \mathrm{H}, \mathrm{H}-3), 6.88-6.93(\mathrm{~m}, 3 \mathrm{H}), 7.09-7.13$ $(\mathrm{m}, 3 \mathrm{H}), 7.37\left(\mathrm{~d}, 1 \mathrm{H}, \mathrm{J}_{0}=8.8, \mathrm{H}-7\right), 11.50(\mathrm{~s}, 1 \mathrm{H}, \mathrm{N}-\mathrm{H}) .{ }^{13} \mathrm{C}-\mathrm{NMR} \delta \mathrm{ppm}$ (DMSO-d $\left.\mathrm{d}_{6}\right)$ : 20.038; 49.155; 55.228; 101.894; 103.845; 112.874; 114.307; 116.151; 127.086; 128.290; 129.433; 130.134; 131.186; 148.621; 153.741; 161.994. MS $(\mathrm{ESI}+) \mathrm{m} / \mathrm{z}: 350.5(\mathrm{M}+\mathrm{H}, 100 \%)$. Anal. Calcd for $\mathrm{C}_{21} \mathrm{H}_{23} \mathrm{~N}_{3} \mathrm{O}_{2} \cdot 0.1 \mathrm{H}_{2} \mathrm{O}: \mathrm{C}, 71.81 ; \mathrm{H}, 6.65 ; \mathrm{N}, 11.96$. Found: C, 71.76; H, 6.69; N, 11.89 .

\subsection{3. (5-Fluoro-1H-indol-2-yl)(4-(4-fluorophenyl)piperazin-1-yl)methanone (3)}

CAS Registry Number: 902025-79-0. Yield: 24\%; mp.: 242-244 ${ }^{\circ} \mathrm{C} .{ }^{1} \mathrm{H}-\mathrm{NMR}\left(400 \mathrm{MHz}, \mathrm{DMSO}-d_{6}\right) ; \delta$ ppm: $3.16(\mathrm{t}, 4 \mathrm{H}$, piperazine- $\mathrm{H}), 3.88\left(\mathrm{bs}, 4 \mathrm{H}\right.$, piperazine-H), $6.82(\mathrm{~s}, 1 \mathrm{H}, \mathrm{H}-3), 6.97-7.10(\mathrm{~m}, 5 \mathrm{H}), 7.37\left(\mathrm{dd}, 1 \mathrm{H}, J_{m}=2.4\right.$ $\left.\mathrm{Hz}, J_{0}=9.6 \mathrm{~Hz}\right), 7.42\left(\mathrm{dd}, 1 \mathrm{H}, J_{m}=4.4 \mathrm{~Hz}, J_{o}=8.8 \mathrm{~Hz}\right), 11.72(\mathrm{~s}, 1 \mathrm{H}, \mathrm{N}-\mathrm{H}) \cdot{ }^{13} \mathrm{C}-\mathrm{NMR} \delta$ ppm (DMSO- $\left.d_{6}\right): 49.376$; 104.054; 105. 467; 111.895; 113.240; 115.366; 117.690; 126.853; 131.574; 132.664; 147.614; 155.128; 155.974; 157.475; 158.283; 161.674. MS (ESI +) m/z: $342.2(\mathrm{M}+\mathrm{H}, 100 \%)$. Anal. Calcd for $\mathrm{C}_{19} \mathrm{H}_{17} \mathrm{~F}_{2} \mathrm{~N}_{3} \mathrm{O}: \mathrm{C}, 66.85 ; \mathrm{H}, 5.01 ; \mathrm{N}, 12.30$. Found: C, 66.62; H, 5.21; N, 12.20 .

\subsection{4. (4-(4-Fluorophenyl)piperazin-1-yl)(5-methoxy-1H-indol-2-yl)methanone (4)}

CAS Registry Number: 878987-70-3. Yield: 28\%; mp.: $172-173{ }^{\circ} \mathrm{C} .{ }^{1} \mathrm{H}-\mathrm{NMR}\left(400 \mathrm{MHz}, \mathrm{DMSO}-d_{6}\right) ; \delta$ ppm: $3.15(\mathrm{t}, 4 \mathrm{H}$, piperazine- $\mathrm{H}), 3.74\left(\mathrm{~s}, 3 \mathrm{H},-\mathrm{OCH}_{3}\right), 3.88(\mathrm{bs}, 4 \mathrm{H}$, piperazine- $\mathrm{H}), 6.73(\mathrm{~s}, 1 \mathrm{H}, \mathrm{H}-3), 6.84\left(\mathrm{dd}, 1 \mathrm{H}, J_{m}=2.4\right.$ $\left.\mathrm{Hz}, J_{0}=8.8 \mathrm{~Hz}, \mathrm{H}-6\right), 6.96-7.00(\mathrm{~m}, 2 \mathrm{H}), 7.05-7.09(\mathrm{~m}, 3 \mathrm{H}), 7.31\left(\mathrm{~d}, 1 \mathrm{H}, J_{0}=8.8 \mathrm{~Hz}\right), 11.45(\mathrm{~s}, 1 \mathrm{H}, \mathrm{N}-\mathrm{H}) .{ }^{13} \mathrm{C}-\mathrm{NMR} \delta$ ppm (DMSO- $d_{6}$ ): 49.391; 55.220; 101.894; 103.867; 112.874; 114.330; 115.358; 117.667; 127.078; 130.096; 131.193; 147.645; 153.749; 155.113; 157.452; 161.994. MS (ESI+) m/z: $354.2(\mathrm{M}+\mathrm{H}, 100 \%)$. Anal. Calcd for $\mathrm{C}_{20} \mathrm{H}_{20} \mathrm{FN}_{3} \mathrm{O}_{2}$ 0.2 $\mathrm{H}_{2} \mathrm{O}: \mathrm{C}, 67.28 ; \mathrm{H}, 5.75 ; \mathrm{N}, 11.77$. Found: $\mathrm{C}, 67.12 ; \mathrm{H}, 5.79 ; \mathrm{N}, 11.71$.

\subsection{5. (5-Fluoro-1H-indol-2-yl)(4-(4-(trifluoromethyl)phenyl)piperazin-1-yl)methanone (5)}

Yield: $24 \%$; mp.: $\left.297-298^{\circ} \mathrm{C} .{ }^{1} \mathrm{H}-\mathrm{NMR}(400 \mathrm{MHz} \text {, DMSO-d })_{6}\right) ; \delta \mathrm{ppm}$ : $3.43(\mathrm{t}, 4 \mathrm{H}$, piperazine-H), 3.90 (bs, $4 \mathrm{H}$, piperazine-H), $6.84(\mathrm{~s}, 1 \mathrm{H}, \mathrm{H}-3), 7.04\left(\mathrm{dd}, 1 \mathrm{H}, J_{0}=9.2 \mathrm{~Hz}, J_{m}=2.4 \mathrm{~Hz}\right), 7.08\left(\mathrm{~d}, 2 \mathrm{H}, J_{0}=8.4 \mathrm{~Hz}, \mathrm{H}-2^{\prime}, 6^{\prime}\right), 7,37$ $\left(\mathrm{dd}, 1 \mathrm{H}, J_{m}=2.8 \mathrm{~Hz}, J_{0}=9.6 \mathrm{~Hz}\right) 7.43\left(\mathrm{dd}, 1 \mathrm{H}, J_{m}=4.8 \mathrm{~Hz}, J_{0}=9.2 \mathrm{~Hz}\right), 7.53\left(\mathrm{~d}, 2 \mathrm{H}, J_{0}=8.8 \mathrm{~Hz}, \mathrm{H}-3^{\prime}, 5^{\prime}\right), 11.69(\mathrm{~s}, 1 \mathrm{H}$, N-H). ${ }^{13}$ C-NMR $\delta$ ppm (DMSO- $\left.d_{6}\right)$ : 46.869; 104.199; 105.490; 111.838; 112.097; 113.266; 114.178; 117.889; 118.201; 123.604; 126.248; 126.868; 131.498; 132.687; 152.796; 155.974; 158.291; 161.758. MS (ESI +) m/z: $392.2(\mathrm{M}+\mathrm{H}$, 100\%). Anal. Calcd for $\mathrm{C}_{20} \mathrm{H}_{17} \mathrm{~F}_{4} \mathrm{~N}_{3} \mathrm{O}$ : C, 61.37; H, 4.37; N, 10.73. Found: C, 61.07; H, 4.44; N, 10.64 . 


\subsection{6. (5-Methoxy-1H-indol-2-yl)(4-(4-(trifluoromethyl)phenyl)piperazin-1-yl)methanone (6)}

Yield: $49 \%$; mp.: 198-200 ${ }^{\circ} \mathrm{C} .{ }^{1} \mathrm{H}-\mathrm{NMR}\left(400 \mathrm{MHz}, \mathrm{DMSO}-d_{6}\right)$; $\delta$ ppm: $3.42(\mathrm{t}, 4 \mathrm{H}$, piperazine-H), 3.75 (s, $\left.3 \mathrm{H},-\mathrm{OCH}_{3}\right), 3.91\left(\mathrm{bs}, 4 \mathrm{H}\right.$, piperazine-H), $6.76\left(\mathrm{~d}, 1 \mathrm{H}, J_{m}=2 \mathrm{~Hz}, \mathrm{H}-3\right), 6.85\left(\mathrm{dd}, 1 \mathrm{H}, J_{m}=2.8 \mathrm{~Hz}, J_{o}=9.2 \mathrm{~Hz}, \mathrm{H}-6\right)$, $7.07\left(\mathrm{~d}, 2 \mathrm{H}, J_{0}=8 \mathrm{~Hz}, \mathrm{H}-2^{\prime}, 6^{\prime}\right), 7.08\left(\mathrm{~d}, 1 \mathrm{H}, J_{m}=2\right), 7.32\left(\mathrm{~d}, 1 \mathrm{H}, J_{o}=8.4 \mathrm{~Hz}, \mathrm{H}-7\right), 7.53\left(\mathrm{~d}, 2 \mathrm{H}, J_{0}=8.4 \mathrm{~Hz}, \mathrm{H}-3^{\prime}, 5^{\prime}\right)$, 11.47 (s, $1 \mathrm{H}, \mathrm{N}-\mathrm{H}$ ). ${ }^{13} \mathrm{C}-\mathrm{NMR} \delta \mathrm{ppm}\left(\mathrm{DMSO}-d_{6}\right.$ ): 46.869; 55.220; 101.894; 104.005; 112.905; 114.147; 114.406; $117.843 ; 118.155 ; 123.604 ; 126.240 ; 127.101 ; 130.027 ; 131.209 ; 152.812 ; 153.749 ; 162.070$. MS (ESI +) m/z: 404.4 $(\mathrm{M}+\mathrm{H}, 100 \%)$. Anal. Calcd for $\mathrm{C}_{21} \mathrm{H}_{20} \mathrm{~F}_{3} \mathrm{~N}_{3} \mathrm{O}_{2}$ : C, 62.52; H, 4.99; N, 10.41. Found: $\mathrm{C}, 62.76 ; \mathrm{H}, 5.34 ; \mathrm{N}, 10.10$.

\subsection{7. (5-Fluoro-1H-indol-2-yl)(4-(4-fluorobenzyl)piperazin-1-yl)methanone (7)}

Yield: $18 \%$; mp.: $192-193{ }^{\circ} \mathrm{C} .{ }^{1} \mathrm{H}-\mathrm{NMR}\left(400 \mathrm{MHz}\right.$, DMSO- $\left.d_{6}\right) ; \delta$ ppm: $2.41(\mathrm{t}, 4 \mathrm{H}$, piperazine-H), 3.49 (s, $\left.2 \mathrm{H},-\mathrm{CH}_{2}-\right), 3.72\left(\mathrm{bs}, 4 \mathrm{H}\right.$, piperazine-H), $6.73\left(\mathrm{~d}, 1 \mathrm{H}, \mathrm{J}_{m}=1.6, \mathrm{H}-3\right), 7.02(\mathrm{t}, 1 \mathrm{H}), 7.11-7.16\left(\mathrm{~m}, 2 \mathrm{H}, \mathrm{H}-3^{\prime}, 5^{\prime}\right), 7.31-$ $7.36(\mathrm{~m}, 3 \mathrm{H}), 7.39\left(\mathrm{dd}, 1 \mathrm{H}, J_{m}=4.8 \mathrm{~Hz}, J_{o}=8.8 \mathrm{~Hz}\right), 11.63(\mathrm{~s}, 1 \mathrm{H}, \mathrm{N}-\mathrm{H}) .{ }^{13} \mathrm{C}-\mathrm{NMR} \delta \mathrm{ppm}\left(\mathrm{DMSO}-d_{6}\right): 52.465 ; 60.820$; 103.849; 105.375; 111.739; 113.144; 114.868; 126.813; 130.677; 131.645; 132.575; 133.899; 155.908; 158.222; 160.063; 161.531; 162.474. MS (ESI +) m/z: $356.5(\mathrm{M}+\mathrm{H}, 100 \%)$. Anal. Calcd for $\mathrm{C}_{20} \mathrm{H}_{19} \mathrm{~F}_{2} \mathrm{~N}_{3} \mathrm{O}: \mathrm{C}, 67.59 ; \mathrm{H}, 5.38 ; \mathrm{N}, 11.82$. Found: C, 67.49; H, 5.37; N, 11.73 .

\subsection{8. (4-(4-Fluorobenzyl)piperazin-1-yl)(5-methoxy-1H-indol-2-yl)methanone (8)}

Yield: 19\%; mp.: 163-165 ${ }^{\circ} \mathrm{C} .{ }^{1} \mathrm{H}-\mathrm{NMR}\left(400 \mathrm{MHz}, \mathrm{DMSO}-d_{6}\right)$; $\delta$ ppm: 2.42 (bs, 4H, piperazine-H), 3.50 (s, $\left.2 \mathrm{H},-\mathrm{CH}_{2}-\right), 3.74\left(\mathrm{~s}, 7 \mathrm{H}\right.$, piperazine- $\mathrm{H}$ and $\left.-\mathrm{OCH}_{3}\right), 6.67\left(\mathrm{~d}, 1 \mathrm{H}, J_{m}=1.6 \mathrm{~Hz}, \mathrm{H}-3\right), 6.83\left(\mathrm{dd}, 1 \mathrm{H}, J_{m}=2.4 \mathrm{~Hz}, J_{o}=8.8\right.$ Hz, H-6), $7.04\left(\mathrm{~d}, 1 \mathrm{H}, J_{m}=2.4 \mathrm{~Hz}, \mathrm{H}-4\right), 7.15\left(\mathrm{t}, 2 \mathrm{H}, \mathrm{H}-3^{\prime}, 5^{\prime}\right), 7.30$ (d, 1H, $\left.J_{0}=8.8 \mathrm{~Hz}, \mathrm{H}-7\right), 7.36$ (m, 2H, H-2', 6'), $11.41(\mathrm{~s}, 1 \mathrm{H}, \mathrm{N}-\mathrm{H}) .{ }^{13} \mathrm{C}-\mathrm{NMR} \delta \mathrm{ppm}\left(\mathrm{DMSO}-d_{6}\right): 52.546 ; 55.213 ; 60.859 ; 101.879 ; 103.730 ; 112.836 ; 114.238$; 114.825; 115.039; 127.063; 130.180; 130.751; 131.132; 153.711; 160.112; 161.887; 162.520. MS (ESI +) m/z: 368.5 $(\mathrm{M}+\mathrm{H}, 100 \%)$. Anal. Calcd for $\mathrm{C}_{21} \mathrm{H}_{22} \mathrm{FN}_{3} \mathrm{O}_{2}: \mathrm{C}, 68.64 ; \mathrm{H}, 6.03 ; \mathrm{N}, 11.43$. Found: $\mathrm{C}, 68.42 ; \mathrm{H}, 6.12 ; \mathrm{N}, 11.38$.

\subsection{9. (4-(4-Chlorophenyl)piperazin-1-yl)(5-methoxy-1H-indol-2-yl)methanone (9)}

Yield: 35\%; mp.: $191-192{ }^{\circ} \mathrm{C} .{ }^{1} \mathrm{H}-\mathrm{NMR}\left(400 \mathrm{MHz}\right.$, DMSO- $\left.d_{6}\right) ; \delta \mathrm{ppm:} 3.21(\mathrm{t}, 4 \mathrm{H}$, piperazine-H), $3.75(\mathrm{~s}$, $\left.3 \mathrm{H},-\mathrm{OCH}_{3}\right), 3.88\left(\mathrm{bs}, 4 \mathrm{H}\right.$, piperazine-H), $6.75\left(\mathrm{~d}, 1 \mathrm{H}, J_{m}=1.2 \mathrm{~Hz}, \mathrm{H}-3\right), 6.85\left(\mathrm{dd}, 1 \mathrm{H}, J_{m}=2.4 \mathrm{~Hz}, J_{o}=8.8 \mathrm{~Hz}, \mathrm{H}-6\right)$, $6.97\left(\mathrm{~d}, 2 \mathrm{H}, J_{0}=9.2 \mathrm{~Hz}, \mathrm{H}-2^{\prime}, 6^{\prime}\right), 7.07\left(\mathrm{~d}, 1 \mathrm{H}, J_{m}=2.4 \mathrm{~Hz}, \mathrm{H}-4\right), 7.26\left(\mathrm{~d}, 2 \mathrm{H}, J_{0}=9.2 \mathrm{~Hz}, \mathrm{H}-3^{\prime}, 5^{\prime}\right), 7.32\left(\mathrm{~d}, 1 \mathrm{H}, J_{0}=8.4\right.$ $\mathrm{Hz}, \mathrm{H}-7), 11.46$ (s, $1 \mathrm{H}, \mathrm{N}-\mathrm{H}) .{ }^{13} \mathrm{C}-\mathrm{NMR} \delta \mathrm{ppm}\left(\mathrm{DMSO}-d_{6}\right)$ : 48.732; 55.714; 102.386; 104.393; 113.376; 114.845; $117.621 ; 123.231 ; 127.572 ; 129.175 ; 130.560 ; 131.689 ; 149.982 ; 154.239 ; 162.504$. MS (ESI +) m/z: $370.4(\mathrm{M}+\mathrm{H}$, $100 \%), 372.5(\mathrm{M}+2,33 \%)$. Anal. Calcd for $\mathrm{C}_{20} \mathrm{H}_{20} \mathrm{ClN}_{3} \mathrm{O}_{2}$ : C, 64.95; H, 5.45; N, 11.36. Found: $\mathrm{C}, 65.11 ; \mathrm{H}, 5.65$; N, 11.38 .

\subsubsection{0. (5-Methoxy-1H-indol-2-yl)(4-(4-nitrophenyl)piperazin-1-yl)methanone (10)}

Yield: 37\%; mp.: 224-226 ${ }^{\circ} \mathrm{C} .{ }^{1} \mathrm{H}-\mathrm{NMR}\left(400 \mathrm{MHz}\right.$, DMSO- $\left.d_{6}\right)$; $\delta$ ppm: 3.61 (s, $4 \mathrm{H}$, piperazine-H), 3.75 (s, $3 \mathrm{H},-\mathrm{OCH} 3), 3.92\left(\mathrm{~s}, 4 \mathrm{H}\right.$, piperazine-H), $6.76(\mathrm{~s}, 1 \mathrm{H}, \mathrm{H}-3), 6.85\left(\mathrm{~d}, 1 \mathrm{H}, J_{0}=8.4 \mathrm{~Hz}\right), 6.98\left(\mathrm{~d}, 2 \mathrm{H}, J_{0}=8.8 \mathrm{~Hz}, \mathrm{H}-2^{\prime}, 6^{\prime}\right)$ $7.07(\mathrm{~s}, 1 \mathrm{H}), 7.33\left(\mathrm{~d}, 1 \mathrm{H}, J_{0}=9.2 \mathrm{~Hz}\right), 8.07\left(\mathrm{~d}, 2 \mathrm{H}, J_{0}=8.8 \mathrm{~Hz}, \mathrm{H}-3^{\prime}, 5^{\prime}\right), 11.38(\mathrm{~s}, 1 \mathrm{H}, \mathrm{N}-\mathrm{H}) .{ }^{13} \mathrm{C}-\mathrm{NMR} \delta \mathrm{ppm}$ (DMSO$\left.d_{6}\right): 45.787 ; 55.205 ; 101.970 ; 104.081 ; 112.227 ; 112.844 ; 114.406 ; 125.638 ; 127.094 ; 129.928 ; 131.201 ; 136.931 ;$ 153.726; 153.168; 162.093. MS (ESI -) m/z: 379.5 (M - H, 100\%). Anal. Calcd for $\mathrm{C}_{20} \mathrm{H}_{20} \mathrm{~N}_{4} \mathrm{O}_{4} \cdot 2 \mathrm{H}_{2} \mathrm{O}: \mathrm{C}, 57.68 ; \mathrm{H}$, 5.8; N, 13.45. Found: C, 57.68; H, 5.90; N, 13.49 .

\subsubsection{1. (5-Fluoro-1H-indol-2-yl)(4-(4-hydroxyphenyl)piperazin-1-yl)methanone (11)}

Yield: $45 \%$; mp.: $234-236{ }^{\circ} \mathrm{C} .{ }^{1} \mathrm{H}-\mathrm{NMR}\left(400 \mathrm{MHz}\right.$, DMSO- $\left.d_{6}\right) ; \delta \mathrm{ppm}: 3.02(\mathrm{t}, 4 \mathrm{H}$, piperazine-H), 3.86 (bs, $4 \mathrm{H}$, piperazine- $\mathrm{H}), 6.67\left(\mathrm{~d}, 2 \mathrm{H}, J_{0}=8.8 \mathrm{~Hz}, \mathrm{H}-2^{\prime}, 6^{\prime}\right), 6.80\left(\mathrm{~d}, 1 \mathrm{H}, J_{m}=1.6 \mathrm{~Hz}, \mathrm{H}-3\right), 6.83\left(\mathrm{~d}, 2 \mathrm{H}, J_{0}=9.2 \mathrm{~Hz}, \mathrm{H}-3^{\prime}, 5^{\prime}\right)$, $7.05\left(\mathrm{td}, 1 \mathrm{H}, J_{m}=2.4 \mathrm{~Hz}, J_{0}=9 \mathrm{~Hz}\right), 7.36\left(\mathrm{dd}, 1 \mathrm{H}, J_{m}=2.4 \mathrm{~Hz}, J_{0}=9.6 \mathrm{~Hz}\right), 7.42\left(\mathrm{dd}, 1 \mathrm{H}, J_{m}=4.8 \mathrm{~Hz}, J_{0}=8.6 \mathrm{~Hz}\right), 8.89$ $(\mathrm{s}, 1 \mathrm{H}, \mathrm{OH}), 11.71(\mathrm{~s}, 1 \mathrm{H}, \mathrm{N}-\mathrm{H}) .{ }^{13} \mathrm{C}-\mathrm{NMR} \delta \mathrm{ppm}\left(\mathrm{DMSO}-d_{6}\right): 28.969 ; 30.363 ; 50.557 ; 103.989 ; 105.456 ; 111.845$; 113.217; 115.496; 118.460; 126.857; 131.658; 132.649; 143.843; 151.417; 155.966; 158.283; 161.636. MS (ESI +) m/z: $340.1(\mathrm{M}+\mathrm{H}, 100 \%)$. Anal. Calcd for $\mathrm{C}_{19} \mathrm{H}_{18} \mathrm{FN}_{3} \mathrm{O}_{2} \cdot 0.5 \mathrm{C}_{6} \mathrm{H}_{14} 0.3 \mathrm{H}_{2} \mathrm{O}: \mathrm{C}, 68.12 ; \mathrm{H}, 6.65 ; \mathrm{N}, 10.83$. Found: $\mathrm{C}, 68.15$; $\mathrm{H}, 6.43 ; \mathrm{N}, 10.49$.

\subsection{In vitro antioxidant activities}

\subsubsection{Superoxide radical scavenging activity (Cytochrome C Assay)}

Superoxide radical scavenging activities of the synthesized compounds was determined according to a previously described method [39]. All experiments were carried out in triplicate and the results were given as 
a percentage of the control. Vit-E was used as a positive control. Superoxide radical scavenging capacity was calculated using the formula given below:

Superoxide radical scavenging activity $(\%)=\left[\left(\mathrm{A}_{\text {control }}-\mathrm{A}_{\text {test }}\right) /\left(\mathrm{A}_{\text {control }}-\mathrm{A}_{\text {blank }}\right)\right] \times 100$

$A_{\text {control }}=$ the absorbance of the control excluding test compounds; $A_{\text {test }}=$ is the absorbance of the test compound; $A_{\text {blank }}=$ the absorbance of the blank excluding test compounds and the superoxide radical generating system.

\subsubsection{DPPH free radical scavenging activity}

The free radical scavenging activity of the compounds was determined using a previously described method with DPPH radicals [40]. Each experiment was carried out in triplicate and a -tocopherol was used as standard. The ability to scavenge DPPH radicals was calculated from the following equation:

$$
\text { DPPH free radical scavenging activity }(\%)=\left[\left(\mathrm{A}_{\text {control }}-\mathrm{A}_{\text {test }}\right) / \mathrm{A}_{\text {control }}\right] \times 100
$$

$\mathrm{A}_{\text {test }}=$ the absorbance of DPPH radical and compounds; $\mathrm{A}_{\text {control }}=$ the absorbance recorded for methanolic solution of DPPH and DMSO solution excluding test compounds.

\subsubsection{Lipid peroxidation}

The effect of the synthesized compounds on rat liver homogenate induced with $\mathrm{FeCl}_{2}$-ascorbic acid, and LP was measured by the method descibed previously [41]. a-Tocopherol was used as a standard. Each experiment was performed in triplicate. Lipid peroxidation inhibitory activity (\%) was calculated from the folowing equation:

$$
\text { LP inhibitory activity }(\%)=\left[\left(\mathrm{A}_{\text {control }}-\mathrm{A}_{\text {test }}\right) /\left(\mathrm{A}_{\text {control }}-\mathrm{A}_{\text {blank }}\right)\right] \times 100
$$

$\mathrm{A}_{\text {control }}=$ the absorbance of the control excluding test compounds; $\mathrm{A}_{\text {test }}=$ the absorbance of the test compounds; $A_{b l a n k}=$ the absorbance of the blank excluding test compounds and the free radical generating sytem ( $\mathrm{Fe}^{+2} /$ ascorbate).

\subsubsection{Anti-inflammatory activity}

Fresh whole human blood was collected from healthy human volunteer who did not take any antiinflammatory or steroidal drug for 2 weeks prior the experiment. The tubes were centrifuged at $3000 \mathrm{rpm}$ for $10 \mathrm{~min}$. The packed cells were washed with equal volume of isosaline $(0.85 \%, \mathrm{pH} 7.2)$. The volume of the blood was measured and reconstituted as $10 \% \mathrm{v} / \mathrm{v}$ suspension with isosaline. The membrane stabilizing activities of the compounds were evaluated using heat induced human erythrocyte hemolysis, designed by Anosike et al and Debnath et al with minor modifications. The reaction mixture consisted of equal volume of test sample and $10 \%$ red blood cells suspension. All the centrifuge tubes containing reaction mixture were incubated at 56 ${ }^{\circ} \mathrm{C}$ for $30 \mathrm{~min}$. At the end of the incubation the tubes were cooled. The reaction mixture was centrifuged at $2500 \mathrm{rpm}$ for $5 \mathrm{~min}$. Then the absorbance of the supernatant was measured at $560 \mathrm{~nm}$. The experiment was performed in triplicates for all the test samples. The results were expressed as the half maximal inhibitory concentration $\left(\mathrm{IC}_{50}\right)[42,43]$. ASA was used as a standard drug. The percentage hemolysis and protection was calculated according to the formula:

Hemolysis $\%=\left(\right.$ Absorbance $_{\text {test sample }} /$ Absorbance $\left._{\text {control }}\right) \times 100$

Protection $\%=100-\left[\left(\right.\right.$ Absorbance $_{\text {test sample }} /$ Absorbance $\left.\left._{\text {control }}\right) \times 100\right]$

\subsection{Molecular docking and molecular properties prediction}

Molecular docking was carried out by using AutodockVina program. X-ray crystal structure of COX-2 (PDB ID: 3NT1) and the relative ligand were downloaded from Protein Data Bank (http://www.rcsb.org). AutoDock-Tools 1.5.6 (ADT) was used for preparing the pdbqt files. The protein was optimized by removing 
water and co-crystallized ligand. Then polar hydrogens were added. Compounds were also energetically minimized. A grid box of $16 \times 16 \times 16 \AA(x, y$, and $z)$ was created around the enzyme active pocket with the spacing of $1 \AA$ and has the average coordinates of the crystallographic ligand in the pdb structure. Exhaustiveness was set to 10 . Other vina docking parameters were set to default. The 3D compound-protein docking posses were analyzed manually using AutoDockTools.

Physicochemical features such as topological surface area, number of rotatable bonds and hydrogen bond acceptors \& donors and Log P were calculated using Mol inspiration online tool [36].

\subsection{Statistical analysis}

The Statistical Package for the Social Sciences (SPSS) version 25.0 was used to perform the statistical analysis. All experiments were done in triplicate and the results were expressed as mean \pm SD. For the data, the analysis of variance was used to determine whether there are any significant differences between the means of the groups (ANOVA, Tukey's test). The differences between the groups were evaluated with Kruskal-Wallis test. $\mathrm{p}<0.05$ was considered statistically significant.

Acknowledgements: We thank Prof. Hakan Goker and Assoc. Prof. Mehmet Alp from Central Instrumentation Laboratory of Faculty of Pharmacy, Ankara University for NMR and elemental analysis.

Author contributions: Concept - T.G.A., Z.K.K.; Design - T.G.A., Z.K.K.; Supervision - T.G.A., Z.K.K.; Resources T.G.A., Z.K.K.; Materials - T.G.A., Z.K.K.; Data Collection and/or Processing - A.B., C.A, Z.K.K, T.Ç., S.Y.S; Analysis and/or Interpretation - Z.K.K., C.A., T.Ç., S.Y.S; Literature Search - Z.K.K., C.A., A.B.; Writing - Z.K.K., C.A.; Critical Reviews - T.G.A., A.B., Z.K.K., C.A., S.Y.S., T.Ç.

Conflict of interest statement: The authors declare no conflict of interest.

Ethics committee approval: Lipid peroxidation protocols were approved by Gazi University Animal Experiments Local Ethics Committee, Ankara-Turkey (02.11.2016/G.Ü.ET-16.067). Anti-inflammatory activity protocol was approved by the ethics committees of the Faculty of Medicine of Ankara University, Ankara-Turkey (26.10.2015/16-695-15).

\section{REFERENCES}

[1] Kumar R, Clermont G, Vodovotz Y, Chow CC. The dynamics of acute inflammation. J Theor Biol. 2004; 230: 145-155. [CrossRef]

[2] Tilg H, Moschen AR. Adipocytokines: mediators linking adipose tissue, inflammation and immunity. Nat Rev Immunol. 2006; 6: 772-783. [CrossRef]

[3] Biswas SK. Does the interdependence between oxidative stress and inflammation explain the antioxidant paradox? Oxid Med Cell Longev. 2016; 5698931. [CrossRef]

[4] Weber V, Rubat C, Duroux E, Lartigue C, Madesclaire M, Coudert P. New 3- and 4-hydroxyfuranones as antioxidants and anti-inflammatory agents. Bioorg Med Chem. 2005; 13: 4552-4564. [CrossRef]

[5] Geronikaki AA, Lagunin AA, Hadjipavlou-Litina DI, Eleftheriou PT, Filimonov DA, Poroikov VV, Alam I, Saxena AK. Computer-aided discovery of anti-inflammatory thiazolidinones with dual cyclooxygenase/lipoxygenase inhibition. J Med Chem. 2008; 51: 1601-1609. [CrossRef]

[6] Suthar SK, Jaiswal V, Lohan S, Bansal S, Chaudhary A, Tiwari A, Alex AT, Joesph A. Novel quinolone substituted thiazolidin-4-ones as anti-inflammatory, anticancer agents: Design, synthesis and biological screening. Eur J Med Chem. 2013; 63: 589-602. [CrossRef]

[7] Yuan T, Yang T, Chen H, Fu D, Hu Y, Wang J, Yuan Q, Yu H, Xu W, Xie X. New insights into oxidative stress and inflammation during diabetes mellitus-accelerated atherosclerosis. Redox Biol. 2019; 20: 247-260. [CrossRef]

[8] Mahat RK, Singh N, Rathore V, Arora M, Yadav T. Cross-sectional correlates of oxidative stress and inflammation with glucose intolerance in prediabetes. Diabetes Metab Syndr. 2019; 13: 616-621. [CrossRef]

[9] Cachofeiro V, Goicochea M, de Vinuesa SG, Oubina P, Lahera V, Luno J. Oxidative stress and inflammation, a link between chronic kidney disease and cardiovascular disease: New strategies to prevent cardiovascular risk in chronic kidney disease. Kidney Int. 2008; 74: S4-S9. [CrossRef]

[10] Halliwell B. Oxidative stress and neurodegeneration: where are we now? J Neurochem. 2006; 97: 1634-1658. [CrossRef] 
[11] Lindqvist D, Dhabhar FS, James SJ, Hough CM, Jain FA, Bersani FS, Reus VI, Verhoeven JE, Epel ES, Mahan L, Rosser $\mathrm{R}$, Wolkowitz OM, Mellon SH. Oxidative stress, inflammation and treatment response in major depression. Psychoneuroendocrinology. 2017; 76: 197-205. [CrossRef]

[12] Ambade A, Mandrekar P. Oxidative stress and inflammation: essential partners in alcoholic liver disease. Int J Hepatol. ArticleID 853175, 2012. [CrossRef]

[13] Tucker PS, Scanlan AT, Dalbo VJ. Chronic kidney disease influences multiple systems: describing the relationship between oxidative stress, inflammation, kidney damage, and concomitant disease. Oxid Med Cell Longev. ArticleID 806358, 2015. [CrossRef]

[14] Biswas SK, De Faria JBL. Which comes first: Renal inflammation or oxidative stress in spontaneously hypertensive rats? Free Radic Res. 2007; 41: 216-224. [CrossRef]

[15] Chadha N, Silakari O. Indoles as therapeutics of interest in medicinal chemistry: Bird's eye view. Eur J Med Chem. 2017; 134: 159-184. [CrossRef]

[16] Estevão MS, Carvalho LC, Ribeiro D, Couto D, Freitas M, Gomes A, Ferreira LM, Fernandes E, Marques MM. Antioxidant activity of unexplored indole derivatives: Synthesis and screening. Eur J Med Chem. 2010; 45: 4869-4878. [CrossRef]

[17] Moore PF, Larson DL, Otterness IG, Weissman A, Kadin SB, Sweeney FJ, Eskara JD, Nagahisa A, Sakakibara M, Carty TJ. Tenidap, a structurally novel drug for the treatment of arthritis: Antiinflammatory and analgesic properties. Inflamm Res. 1996; 45: 54-61. [CrossRef]

[18] Ferreira SH, Moncada S, Vane JR. Indomethacin and aspirin abolish prostaglandin release from the spleen. Nat New Biol. 1971; 231: 237-239. [CrossRef]

[19] Simon LS. Actions and toxicity of nonsteroidal anti-inflammatory drugs. Curr Opin Rheumatol. 1996; 8: 169-175. [CrossRef]

[20] Wada Y, Nakamura M, Kogo H, Aizawa Y. Inhibitory effect of acemetacin, a prodrug of indomethacin, on prostaglandin E2 release from inflamed synovial tissue. Jpn J Pharmacol. 1984; 34: 468-470. [CrossRef]

[21] Demerson CA, Humber LG, Abraham NA, Schilling G, Martel RR, Pace-Asciak C. Resolution of etodolac and antiinflammatory and prostaglandin synthetase inhibiting properties of the enantiomers. J Med Chem. 1983; 26: 17781780. [CrossRef].

[22] Costa D, Gomes A, Reis S, Lima JLFC, Fernandes E. Hydrogen peroxide scavenging activity by non-steroidal antiinflammatory drugs. Life Sci. 2005; 76: 2841-2848. [CrossRef]

[23] Reiter RJ, Tan DX, Cabrera J, D'Arpa D, Sainz RM, Mayo JC, Ramos S. The oxidant/antioxidant network: Role of melatonin. Biol Signals Recept. 1999; 8: 56-63. [CrossRef]

[24] Elisabetsky E, Costa-Campos L. The alkaloid alstonine: A review of its pharmacological properties. Evid Based Complement Alternat Med. 2006; 3: 39-48. [CrossRef]

[25] Kruk I, Aboul-Enein HY, Michalska T, Lichszteld K, Kubasik-Kladna K, Ölgen S. In vitro scavenging activity for reactive oxygen species by N-substituted indole-2-carboxylic acid esters. J Lumin. 2007; 22: 379-386. [CrossRef]

[26] Andreadou I, Tsantili-Kakoulidou A, Spyropoulou E, Siatra T. Reactions of indole derivatives with cardioprotective activity with reactive oxygen species. Comparison with melatonin. Chem Pharm Bull. 2003; 51: 1128-1131. [CrossRef]

[27] Aboul-Enein HY, Kladna A, Kruk I, Lichszteld K, Michalska T, Olgen S. Scavenging of reactive oxygen species by novel indolin-2-one and indoline-2-thione derivatives. Biopolymers. 2005; 78: 171-178. [CrossRef]

[28] Suzen S, Bozkaya P, Coban T, Nebioglu D. Investigation of the in vitro antioxidant behaviour of some 2-phenylindole derivatives: discussion on possible antioxidant mechanisms and comparison with melatonin. J Enzym Inhib Med Chem. 2006; 21: 405-411. [CrossRef].

[29] Misik V, Ondrias K, Stasko A. EPR spectroscopy of free radical intermediates of antiarrhythmic-antihypoxic drug stobadine, a pyridoindole derivative. Life Sci. 1999; 65: 1879-1881. [CrossRef]

[30] Cano A, Alcaraz O, Arnao MB. Free radical-scavenging activity of indolic compounds in aqueous and ethanolic media. Anal Bioanal Chem. 2003; 376: 33-37. [CrossRef]

[31] Sharath V, Kumar HV, Naik N. Synthesis of novel indole based scaffolds holding pyrazole ring as anti-inflammatory and antioxidant agents. J Pharm Res. 2013; 6: 785-790. [CrossRef]

[32] Suzen S. Melatonin and synthetic analogs as antioxidants. Curr Drug Delivery. 2013; 10: 71-75. [CrossRef] 
[33] Altuntas TG, Yılmaz N, Çoban T, Ölgen S. Synthesis and antioxidant activity of indole derivatives containing 4substituted piperazine moieties. Lett Drug Des Discov. 2017; 14: 380-386. [CrossRef]

[34] Bhandari K, Murti V, Aruna J, Padam C, Anand N. Agents acting on the central nervous system: Part XXXIII. Synthesis of 1,2,3,4,6,7,8,12-octahydropyrazino $\left[2^{\prime}, 1^{\prime}: 2,1\right]$ pyrido[4,3-b]indole and some 2-substituted aminoalkylindoles. Ind J Chem Sect B. 1979; 17B(3): 246-249.

[35] Duggan KC, Walters MJ, Musee J, Harp JM, Kiefer JR, Oates JA, Marnett LJ. Molecular basis for cyclooxygenase inhibition by the non-steroidal anti-inflammatory drug naproxen. Biol Chem. 2010; 285: 34950-34959. [CrossRef]

[36] Molinspiration Cheminformatics, Bratislava, Slovak Republic. http:/ / www.molinspiration.com/services/properties.html (accessed on 18 August 2019).

[37] Lipinski CA, Lombardo L, Dominy BW, Feeney PJ. Experimental and computational approaches to estimate solubility and permeability in drug discovery and development settings. Adv Drug Deliv Rev. 2001; 46: 3-26. [CrossRef]

[38] Zhao YH, Abraham MH, Lee J, Hersey A, Luscombe CN, Beck G, Sherborne B, Cooper I. Rate-limited steps of human oral absorption and QSAR studies. Pharm Res. 2002; 19: 1446-1457. [CrossRef]

[39] Mc Cord JM, Fridovich I. Superoxide dismutase, an enzymic function for erythrocuprein (Hemocuprein). J Biol Chem. 1969; 243: 6049-6055.

[40] Xie J, Schaich KM. Re-evaluation of the 2,2-diphenyl-1-picrylhydrazyl free radical (DPPH) assay for antioxidant activity. J Agric Food Chem. 2014; 62: 4251-4260. [CrossRef]

[41] Mihara M, Uchiyama M, Fuzukawa K. Thiobarbituric acid value on fresh homogenate of rat as a parameter of lipid peroxidation in aging, CCl4 intoxication, and vitamin E deficiency. Biochem Med. 1980; 23: 302-311. [CrossRef]

[42] Anosike CA, Obidoa O, Ezeanyika LU. Membrane stabilization as a mechanism of the anti-inflammatory activity of methanol extract of garden egg (Solanum aethiopicum). Daru. 2012; 20: 76-83. [CrossRef]

[43] Debnath PC, Das A, Islam A, Islam MA, Hassan MM, Uddin SMG. Membrane stabilization - A possible mechanism of action for the anti-inflammatory activity of a Bangladeshi medicinal plant: Erioglossum rubiginosum (Bara Harina). Pharmacogn J. 2013; 5: 104-107. [CrossRef] 